\title{
Zur Baugeschichte der beiden Wiener Universitätssternwarten
}

\author{
Maria G. Firneis \\ Insitut für Astronomie der Universität Wien, \\ Türkenschanzstraße 17, A-1180 Wien, Austria
}

\begin{abstract}
The first institutionalized university observatory was set up on the roof of the "new" University building in 1755, formally opened 1756. When the observatory's stability was questioned a transfer to the surroundings of Vienna was sought. Various places were tested but finally the former ramparts of the Turks during their sieges of Vienna in the village of Währing were chosen. In 1883 the Austrian Emperor Francis Joseph I held the opening ceremony of this second observatory.

Instrumentation came from the shops of Howard Grubb (Ireland), Clark (Boston), Reichenbach and Merz. Today the building is mainly used as administration and training center for students.
\end{abstract}

Vorgeschichte

Dort wo die Jesuitenkirche in Wien emporragt, befand sich seit Gründung der Universität Wien (formal 1365, zweite Gründung 1385) der zentrale Teil des Universitätsviertels, des "Quartier Latin". Es war das Collegium ducale, das Erzherzog Albrecht der Universität Wien zur Verfügung stellte. Bis heute (es stehen eigentlich nur mehr die Grundmauern) gehören Teile davon dem Jesuitenorden. Zur Zeit der Gründung der Wiener Universitätssternwarte befanden sich Dienst (und Diener)wohnungen von Universitätsprofessoren darinnen.

Die Sternwarte am Dach des Universitätsgebäudes im Stadtzentrum

Als die Habsburgerin Erzherzogin Maria Theresia (Königin von Ungarn und Böhmen, Gattin von Franz Stephan I, Kaiser des hl. Römischen Reiches deutscher Nationen) im Zuge einer Universitätsreform eine neue Aula für die Wiener 
Universität durch Jean Nicolas Jardot [1] errichten ließ, war gerade der kaiserliche Mathematiker und Astronom Johann Jakob de Marinoni verstorben, der eine umfangreiche Sammlung astronomischer Instrumente durch kaiserliche Sponsorenschaft besaß. Maria Theresia ordnete daher den Bau einer Sternwarte ähnlich wie sie der kaiserliche Hofmathematiker Marinoni in seinem Werk "Specola domestica" beschrieben hatte, auf dem Dach des neuen Universitätszentralgebäudes (heute Ignaz Seipel-Platz 2) an.

Dass dadurch die Proportionen des neuen Gebäudes beeinträchtigt wurden, dürfte den Architekten schon einigermaßen gestört haben. Es handelte sich dabei um einen 2 Stock hohen Quertrakt, dessen Zugang über ein verwinkeltes Stiegenhaus gestaltet wurde, das kaum Platz für die notwendige Büchersammlung bot, die P. Maximilian Hell (Jesuit 1720 -1792) dort sofort etablierte. Der medizinische Trakt der Universität mit dem anatomischen Theater (=dem Seziersaal) lag unmittelbar davor, sodaß es nicht ausbleiben konnte, dass der Studentenulk gelegentlich ausuferte und Leichenteile auf dem darüber befindlichen, schlecht zugänglichen Dach landeten, wo sie den Astronomen ungebührlich in die Nase stanken. In Verbindung mit den Erschütterungen der nachts über das Stöckelpflaster vorüberfahrenden Pferdekutschen und den Schallemissionen der mächtigen Glocken der Jesuitenkirche war die Stabilität der Instrumentenaufstellung an dieser Sternwarte ungenügend und es ist daher verständlich, dass die Astronomen über den Standplatz der Sternwarte nicht sehr glücklich waren. Allerdings befand sich in unmittelbarer Nähe dieses Observatoriums auch die eigentliche Jesuitensternwarte und solange der Orden Bestand hatte (Auflösung in Österreich 1773) war damit ein beobachtungstechnischer Vorteil gegeben.

Als J. J. Littrow die Leitung der Universitätssternwarte übernahm war es ihm daher ein wesentliches Anliegen den Beobachtungsstandort zu verlegen, um den Behinderungen der Wiener Innenstadt zu entgehen. Allerdings ließ sich sein Plan nicht durchführen, das Revulotionsjahr 1848 brachte noch zusätzliche Beschränkungen. Zwar durften die Astronomen zu Beobachtungen weiter im Gebäude verbleiben, die Schikanen beim Betreten und Verlassen des Gebäudes waren allerdings erheblich. Trotzdem gelang es Littrow die drei Beobachtungstürme mit drehbaren Kuppeln ausstatten zu lassen, die von einem lokalen Schlosser gefertigt wurden. Der Zugang zu diesen Kuppeln war hingegen als abenteuerlich einzustufen und führte über 3 Giebel des heutigen Akademiegebäudes hinweg, bei Eis und Schnee eine halsbrecherische Tour.

Die Positionsbeobachtungen von Kleinplaneten in Zusammenarbeit mit der Pariser Sternwarte bildete dabei einen wesentlichen Arbeitsschwerpunkt.

Auch unter J.J. Littrows Nachfolger, C. L. Littrow blieb die Verlegung der Sternwarte aus dem Zentrum von Wien ein wesentliches Unterfangen. Anläßlich der Sonnenfinsternis vom 8. Juli 1842 war bereits die Gloriette von 


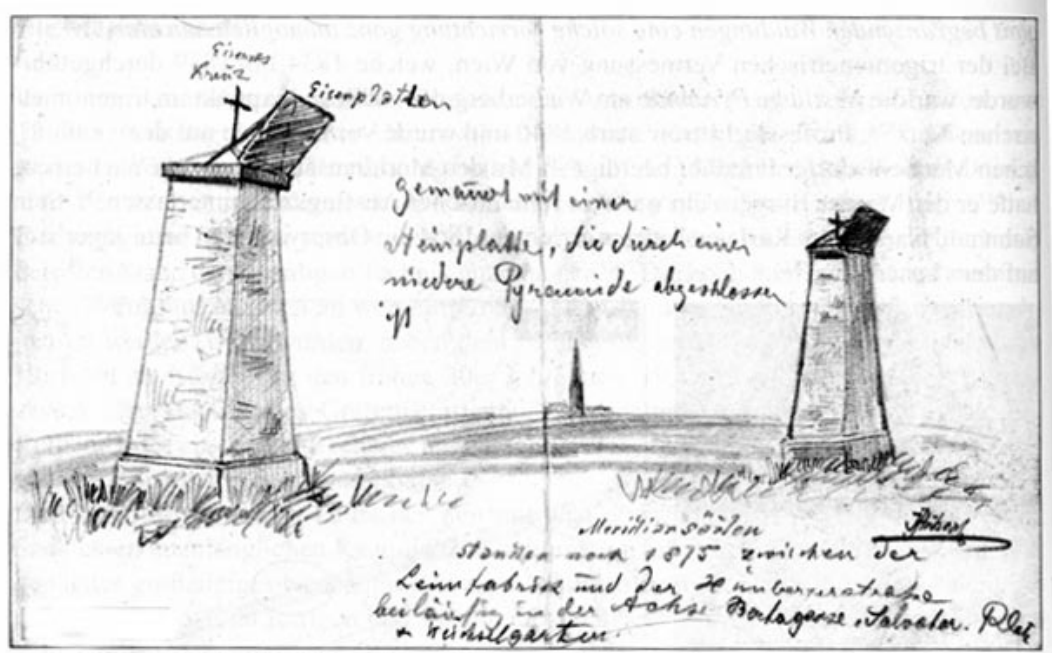

Die Meridiansäulen nach einer Gedächtnis-Skizze (um 1925)

Abb. 1: Meridiansäulen, Wien 10 Schleiergasse [2]

Schloß Schönbrunn als Beobachtungsplatz getestet worden. 1866 wurde eine weitere Teststation am Laaerberg südlich von Wien in Holzbauweise errichtet. Die im Süden dieser Sternwarte gelegenen Meridiansäulen sind sogar noch in einem alten Stadtplan (1902) westlich der Staßenbahnhaltestelle Schleiergasse [2] fälschlicherweise als Bildstöcke eingetragen.

\section{Die Sternwarte in Währing}

Kurzzeitig war sogar das Dach des neuen Universitätsgebäudes am Dr. KarlLueger-Ring als Platz für das astronomische Observatorium diskutiert worden, wogegen sich aber die Astronomen erfolgreich zur Wehr setzten. Das nächste Projekt sah den Höhenrücken Währings Richtung Hernals (heute Kreuzgasse) vor, dann aber wandte sich C.L. Littrow dem Weingarten des Künstlers Ignaz Spöttl (Zeichner und Maler) zu, der ein 5,5ha großes Anwesen in der Ortschaft von Währing mit einem Weinhauerhaus besaß. Schon zuvor war der Höhenrücken von Währing südlich der Türkenschanze in Aussicht genommen worden, allerdings handelte es sich um miliärisches Sperrgebiet (Schießstätte) auf dem Bauverbot herrschte. Dieses wurde bald von reichen Wiener Handelsherren zu Fall gebracht, die ihre Villen im von Heinrich Ferstel und Carl v. Borkowski im Stil eines englischen Cottages geplanten Wohnviertels errichten 


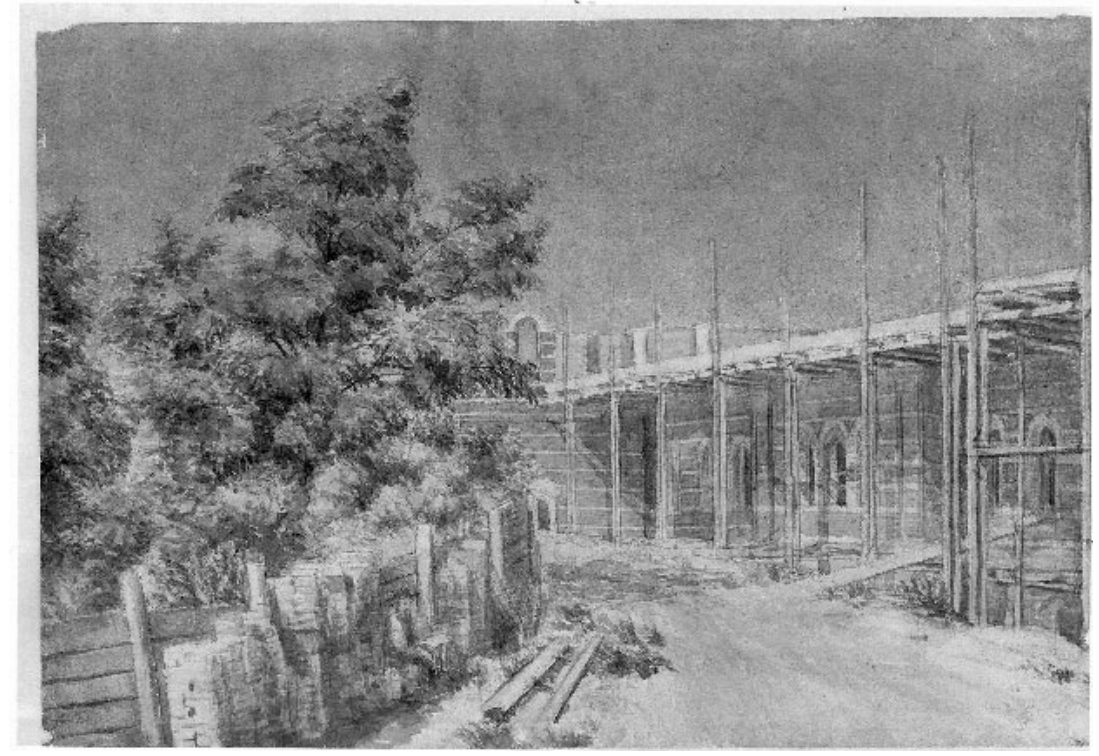

Abb. 2: Bauphase, Bildarchiv Museum Währing

ließen. Das für die Sternwarte in Aussicht genommene Areal wurde den Teilnehmern der Tagung der Astronomischen Gesellschaft im September 1869 vorgestellt, von diesen als passend approbiert und schließlich um 169000 Gulden angekauft. Von 1874-1880 verlief die Bauphase der heutigen Universitätssternwarte, die nach Plänen der Architekten Fellner und Helmer (fußend auf einen Vorläuferplan von Schaller) errichtet wurde. Neben dem Einfluß der Schinkelschen Sternwarte in Berlin war es die Sternwarte von Turku [3] (Finnland) die diesen Bau prägte. Nach mehreren Planänderungen wurde so ein "Palazzo der Sterne" geschaffen, der sowohl einen bemerkenswerten Wohntrakt in Verbindung mit dem eigentlichen Observatoriumstrakt (eine zentrale Hauptkuppel, 3 Seitenkuppeln) in Form eines lateinischen Kreuzes darstellt. Die Dimensionen (Länge, Breite) entsprachen praktisch jenen des Stephansdomes, nur die Höhe der zentralen Kuppel war um $100 \mathrm{~m}$ niederer. Das imposante Stiegenhaus weist bis heute die Handschrift der Architekten auf, die vor allem als Theaterbaumeister (44 Theater Neubauten oder Renovierungen innerhalb der k.k. Monarchie) bekannt waren.

Ausführender Baumeister war Ferdinand Oberwimmer, der sich gleichzeitig im Cottage von Währing eine in ähnlicher Bauweise konzipierte Villa errichtete (was zu bissigen Kommentaren der Anrainer führte). Die Kuppeln der Stern- 
warte wurden von Howard Grubb in Irland gefertigt, der auch das Hauptinstrument herstellte, über Pola nach Wien geliefert und hier von Schlossermeister Ignaz Gridl zusammengesetzt.

Auf dem Sternwarteareal befand sich das "übelbeleumundete" Gasthaus "zum König Sobiesky" (demoliert 1911), welches besonders von Bauarbeitern frequentiert wurde, in dem am 13. April 1875 die Wirtsleute Joseph und Aloisa Schieder ermordet und beraubt wurden.[4]

Carl. L. Littrow hatte die Beendigung des Baus der Sternwarte nicht mehr erlebt. Es war sein Nachfolger Edmund Weiss der am 5. Juni 1883 die feierliche Eröffnung durch Kaiser Franz Joseph I ausrichtete. Weiss hatte sich vor allem in den USA einige Sternwarten angesehen und nach dem Beispiel der innerstädtischen Sternwarte von Washington D.C. Baumpflanzungen im Areal veranlaßt, die die Seeing-Bedingungen optimieren sollten. Ein im selben Jahr errichtetes Portierhäuschen (abgerissen 1976) sollte Sicherheit und Zugang des Geländes kontrollieren.

Obgleich die Wiener Astronomen gerne ein Hauptinstrument der Gebrüder Clark gehabt hätten, wurde durch das Cultus-Ministerium der Konstruktionsauftrag an den irischen Instrumentenbauer Howard Grubb vergeben, der im Analogon zum Washingtoner Instrument, einen 26Zoll Refraktor fertigen sollte. Der von Grubb aus Paris (Fa. Feil) angeforderte Objektivrohling wies allerdings eine Schliere auf, sodaß eine weitere Linse gegossen werden mußte, die Grubb zu einem 27 Zöller schliff (damit war der "Große Refraktor" kurzfristig das weltgrösste Instrument dieses Types). Nach der Schaustellung bei einer Weltausstellung, wurde der "Große Refraktor" wegen Produktionsschwierigkeiten erst 1883 in Wien aufgestellt (Öffnung $68 \mathrm{~cm}$, Brennweite $10,5 \mathrm{~m}, \mathrm{Ge}-$ samtgewicht $13 \mathrm{t}$, Gewicht der beweglichen Teile: 5,5t). Allerdings war das Instrument ausschließlich für den visuellen Bereich konzipiert, sodaß eine spätere photographische Verwendung eine Zeit lang große Schwierigkeiten machte, da Blau- und Gelb-Fokus weit auseinander lagen.

Im Westsaal befand sich ein Meridiankreis von Reichenbach und ein Passageinstrument, das bis in die 60er-Jahre des vergangenen Jahrhunderts in Verwendung stand. In der Westkuppel fand ein $30 \mathrm{~cm}$ Refraktor $(5,2 \mathrm{~m}$ Brennweite) von Clark (Boston) seine Aufstellung. Der Ostmeridiansaal erhielt aus Kostengründen niemals ein Instrument. In der Ostkuppel war ursprünglich ein $15 \mathrm{~cm}$ Frauenhofer-Refraktor (das ehemalige Hauptinstrument der alten Universitätssternwarte) montiert. Heute befindet sich dort ein $20 \mathrm{~cm}$ Refraktor von Starke und Kammer[5].

Der Nordarm des Observatoriums enthielt ursprünglich einen Saal im 1. Vertikal mit einem Spalt öffenbar (heute Hörsaal) in dem kleinere Instrumente zur Anwendung gelangten. In der Nordkuppel war urspünglich ein Kometensucher von Merz (16,2 cm Öffnung) aufgestellt. Später ein $40 \mathrm{~cm}$ Spiegelteleskop 
von Bernhard Schmidt (das Abschiedsgeschenk der Hamburger Sternwarte an Kasimir Graff, als dieser nach Wien ging).

Heute befindet sich dort ein $80 \mathrm{~cm}$ Spiegelteleskop der Firmen "Astro Optik" und "Astro Technik", das mit einem CCD-Photometer ausgestattet ist. Der nördlich an das Sternwartegebäude angrenzende Park hieß ursprünglich Meridianpark. Die in Verlängerung der beiden Meridiansaalspalte verlaufenden Straßenzüge sowie der Park wurden durch ein Servitut zu Gunsten der Universitätssternwarte vor der Verbauung geschützt[6]. Insgesamt betrugen die Baukosten fast 1000000 Goldgulden.

Zwei weitere Gebäude: für ein Coudé-Fernrohr und einen Normalastrographen wurden dank der Sponsorenschaft des Barons Albert v. Rothschild am Gelände errichtet.

\section{Literatur}

[1] R.Wagner-Rieger: Das Haus der Österreichischen Akademie der Wissenschaften, Böhlau, Wien, 1972

[2] W.Sturm: ". . außer der Linie", Favoriten am Wienerberg; Favoritner Museumsschriften, 30, 2004

[3] M.Vyoral-Tschapka: Die Wiener Universitätssternwarte, Österreichische Zeitschrift für Kunst und Denkmalpflege, 1996 H.1/2, Wien

[4] Ein Heimatbuch des 18ten Gemeindebezirkes, Wien, 1925

[5] M.Firneis, E.Göbel: Konzept für öffentliche Führungen - Vienna Internal Report, 1983/2

[6] M.Griebl, M.Firneis: Die Wiener Universitätssternwarte, in Unser Währing

(Vierteljahresheft des Museumsvereines Währing), 43.Jg., 3.Heft, Wien, 2008 